\title{
Structural Derivative Model for Tissue Radiation Response
}

\author{
J. Weberszpil ${ }^{\star}$ and O. Sotolongo-Costa ${ }^{+}$ \\ *Programa de Pós Gradiuação em Modelagem Matemática e Computacional - PPGMMC, Universidade Federal \\ Rural do Rio de Janeiro, UFRRJ-IM/DTL \\ Av. Governador Roberto Silveira s/n- Nova Iguaçú, Rio de Janeiro, Brasil, 695014. \\ josewebe@gmail.com \\ ${ }^{+}$Centro de Investigación en Ciencias, (IICBA), Universidad Autónoma del Estado de Morelos \\ Av. Universidad 1001, 62209 Cuernavaca, Morelos, México. \\ osotolongo@uaem.mx
}

ABSTRACT

By means of a recently-proposed metric or structural derivative, called scale- $q$-derivative approach, we formulate differential equation that models the cell death by a radiation exposure in tumor treatments. The considered independent variable here is the absorbed radiation dose $D$ instead of usual time. The survival factor, $\mathrm{F}_{\mathrm{S}}$, for radiation damaged cell obtained here is in agreement with the literature on the maximum entropy principle, as it was recently shown and also exhibits an excellent agreement with the experimental data. Moreover, the well-known linear and quadratic models are obtained. With this approach, we give a step forward and suggest other expressions for survival factors that are dependent on the complex tumor structure.

\section{Indexing terms/Keywords}

Metric or Structural Derivatives, Scale-q-derivative, Survival factor for tissues irradiation, Radiobiological models, Mathematical modeling tumor,

\section{Academic Discipline And Sub-Disciplines}

Physics and Applied Mathematics/Medical and Biological Physics

\section{SUBJECT CLASSIFICATION}

Theoretical: Applied athematics and Applied Physics

\section{TYPE (METHODIAPPROACH)}

Theoretical: Mathematical Methods in Physics and Medical Physics- Structural or Metric derivatives.

\section{INTRODUCTION}

Recently, one of the authors (J.W.) demonstrated the existence of a possible relationship between $q$-deformed algebras in two different contexts of Non-extensive Statistical Mechanics (NESM), namely, the Tsallis' framework and the Kaniadakis' scenario, with local form of fractional-derivative operators denned in fractal media, the so-called Hausdorff derivatives, mapped into a continuous medium with a fractal measure [1].

In addition, to describe complex systems, the $q$-calculus, in a NESM context, has its formal development based on the definition of deformed expressions for the logarithm and exponential [2], namely, the $q$-logarithm and the $q$-exponential. In such a context, an interesting algebra emerges and the formalism of a deformed derivative opens new possibilities for the treatment of complex systems outside of the thermodynamical spectrum, especially those with fractal or multi-fractal metrics and presenting long-range dynamical interactions. The deformation parameter or entropic index, $q$, has an important place in the description of these complex systems, and describes deviations from standard Lie symmetries. It also provides the formalism to accommodate scale invariance to the thermodynamic formalism, for systems with multi fractal properties. For $q \rightarrow 1$, the formalism reverts to the standard one [2]. In this context, one of the authors (O.S.) has shown, in a recent article, that radiobiological models can be derived, considering the Tsallis' NESM, from the maximum entropy principle and using a cutoff condition motivated by experimental clinical results, with excellent agreement $[3,4]$.

It is our belief that new conceptions and approaches, such as the fractional derivatives (non local operators) and the structural derivatives (local operators; also called deformed or metric or fractal derivatives) [5,6], may allow us to understand new systems, while also helping us to extend well-known results. In particular, regarding to the problem studied, the use of structural derivatives, similarly to the fractional calculus (FC), allows us to describe and emulate complex dynamics involving multiple species and environmental variation, without the addition of explicit terms relating to this complexity in the dynamical equations describing the system.

Here, we set up a formalism that may yield an effective theory to model cell radiation absorption, without the use of statistical averages and without formally imposing any specific nonstandard statistics. For this purpose, we apply the mathematical tool of structural derivatives [5] that includes $q$-derivatives-like, called scale- $q$-derivative [7] and may also be thought with other kind of structural derivatives like Hausdorff derivatives [8, 9, 10] and conformable derivatives [11].

Hence, structural derivatives and/or FC may be the tools to describe, in a softer manner, connections between a coarsegrained medium and dissipation at a certain energy scale. 
We claim that the use of deformed or structural derivatives may enable us to consider the effects of internal times of systems or different internal absorption rates, such as an ecological time proposed in Ref. [12]. This makes sense in the context of complex systems similar to the open species-environmental medium interaction. The approach considered here, seems to be applicable to power-law phenomena, mathematical modeling tumor, biological and logistic growth [13 21], and other dynamical systems.

\section{COMMENTS ON THE USE OF STRUCTURAL DERIVATIVES}

We also suggested that the understanding of the results for the anomalous radiation absorption may be thought in the realm of complexity, mean life, coarse-graininess, and pseudoparticle concepts. Tumor tissue is in fact, complex coarsegraininess structures with internal structure and interacting with media and the radiation.

In a coarse-grained space, a point is not infinitely thin, and here, this feature is modeled by means of a space in which the generic differential is not $d x$, but rather $(d x)^{\alpha}$.

The great majority of actual classical systems is nonconservative but, in spite of that, the most advanced formalisms of classical mechanics deal only with conservative systems [22]. Dissipation [23], for example, is present even at the microscopic level. There is dissipation in every non-equilibrium or fluctuating process, including dissipative tunneling [24] and electromagnetic cavity radiation [25], for instance. In [23], we adopt that a way to suitably treat nonconservative systems is through fractional Calculus (FC), since it can be shown that, for example, a friction force has its form stemming from a Lagrangian that contains a term proportional to the fractional derivative, which may be a derivative of any noninteger order [22, 26-28].

Since we are dealing with open systems, as we already commented, particles or, in the case here, tumor cells constituting the tissue to be irradiated, should in fact be seen as dressed entities or pseudoparticles that exchange energy with other particles and the environment. The system composed by particles and their surroundings may be considered nonconservative due to the possible energy exchange. This energy exchange may be responsible for the resulting noninteger dimension of space-time, giving rise then to an effective coarse-grained medium. Notice that this kind of approach is suitable to treat systems with dissipative forces or non-holonomic systems since it include the scale in time letting to consider the effects of internal times of the systems. Since the tissue radiation absorption is a nonconservative process, all of the mentioned remarks are valid here.

Also, we can make an attempt to connect anomalous diffusion with the anomalous radiation tissue absorption. This connection also re-enforces the use of alternative mathematical approaches. As the diseased tissue is exposed by radiations doses, it changes the way it process the biochemical interactions, including nutrient and gases diffusion or abortion, cell membrane permeability, excretion of metabolites, and so on. The disorder caused by radiation induces a level of disorder in the dynamical processes, with consequent anomalous diffusion like behavior. Since the disorder here is local, the approach with a deformed local operator seems to be easier than the approach of FC used in the study of anomalous diffusion.

It is our belief that new conceptions and approaches, such as metric derivatives and fractional derivatives, may allow us to understand new systems, while also helping us to extend well-known results. In particular, with regards to the studied problem, the use of deformed derivatives, similarly to the FC, allows us to describe and emulate complex dynamics involving multiple tissues and environmental variation, without the addition of explicit terms relating to this complexity in the dynamical equations describing the system. In some way, the formalism proposed here may yield an effective theory for interacting cell-radiation or anomalous growth/death, without the use of statistical averages. Hence, metric derivatives may be the tools to describe, in a softer manner, connections between a coarse-grained medium and dissipation at a certain energy scale.

Some additional support to this view can be found in a recent article that considers a thermodynamics formulation with fractal structure [30]. There, the author considered a Hausdorff dimension and determined the Lipshitz-Hölder exponent in terms of the entropic index $q$, connecting the thermofractal concept - considered a class of thermodynamical systems that present a fractal structure in its thermodynamical description - with hadrons. It is Interesting to observe that the referred author also considered the internal energy of subsystems in such a way that they behave as particles with an internal structure.

\section{METHODS}

In this section, we present some mathematical aspects concerning three different kinds of structural derivative, the Hausdorff derivative, for connections and clarifying reasons, the q- derivative in the context of NESM and the recently proposed scale-q- derivative [7].

\section{Hausdorff derivative}

A model that maps hydrodynamics continuum flow in a fractal coarse-grained (fractal porous) space, which is essentially discontinuous in the embedding Euclidean space, into a continuous flow governed by conventional partial differential equations was suggested in Ref. [31]. In a latter work, Balankin and Espinoza [8] suggested that the discontinuous fractal flow in a fractally permeable medium can be mapped into a fractal continuous flow, which is describable within a continuum framework, indicating also that the geometric framework of fractal continuum model is the three-dimensional 
Euclidean space with a fractal metric. For more details, we refer the interested reader to [8, 9]. Employing the structural differential operators [5] in connection with the Hausdorff derivative [10], one can describe the latter as [9]:

$$
\frac{d^{H}}{d x^{\varsigma}} f(x)=\lim _{x \rightarrow x^{\prime}} \frac{f\left(x^{\prime}\right)-f(x)}{\left(x^{\prime}\right)^{\varsigma}-x^{\varsigma}}=\left(\frac{x}{l_{0}}+1\right)^{1-\varsigma} \frac{d}{d x} f=\frac{l_{0}^{\varsigma-1}}{c_{1}} \frac{d}{d x} f=\frac{d}{d^{\varsigma} x} f,
$$

where $I_{0}$ is the lower cutoff along the Cartesian $x$-axis and the scaling exponent, $\zeta$, characterizes the density of states along the normal direction to the intersection of the fractal continuum with the plane, as defined in Ref. [9].

\section{q- Derivative in the non-extensive context}

Over the recent decades, diverse formalisms have emerged that are adopted to approach complex systems. Among those, we may quote the q-calculus in the Tsallis' version of NESM, with its undeniable success whenever applied to a wide class of different systems; Kaniadakis' approach, based on the compatibility between relativity and thermodynamics; fractional calculus (FC), that deals with the dynamics of anomalous transport and other natural phenomena, and also some local versions of FC that claim to be able to study fractal and multi-fractal spaces and to describe dynamics in these spaces by means of fractional differential equations.

The non-extensive behavior for certain physical systems are in close relation with the presence of spatial-temporal longrange interactions [32]. It is now well known that, for systems as those with gravitational interaction or those where the range of interactions is comparable to system size, with the presence of memory effects, with fractal, multifractal structures, and so on, the standard statistical mechanics and thermodynamics seems not to be the adequate one to describe the dynamics. So, Tsallis [33] has proposed a generalization called nonextensive statistical mechanics and thermodynamics to explain many of the so called anomalous phenomena in the context of complexity. We claim that this may include biological and similar systems, where complexity is ubiquitous [3, 34].

In close relation with NESM statistics, the $q$-derivative sets up a deformed algebra and takes into account that the $q$ exponential is an eigenfunction of $D_{(q)}$ [2]. Borges proposed the following operator for $q$-derivative:

$$
D_{(q)} f(t)=\lim _{y \rightarrow x} \frac{f(x)-f(y)}{x \Theta_{\mathrm{q}} y}=[1+(1-q) x] \frac{d f(x)}{d x} .
$$

Here, $\Theta_{\mathrm{q}}$ is the deformed difference operator, $x \Theta_{\mathrm{q}} y=\frac{x-y}{[1+(1-q) y]},(y \neq 1 /(q-1))$.

The $q$-Integral has the similar structure of Riemann Improper Integral [2]:

$$
\int_{a}^{t} f(x) d_{q} x=\int_{a}^{t} \frac{f(x)}{[1+(1-q) x]} d x
$$

$d_{q} x=\lim _{y \rightarrow x} x \Theta_{\mathrm{q}} y=\frac{1}{[1+(1-q) x]} d x$.

There is also a dual derivative operator $D^{(q)}$, associated with $D_{(q)}$, defined by [2]

$$
D^{(q)} f(t)=\lim _{y \rightarrow x} \frac{f(x) \Theta_{\mathrm{q}} f(y)}{x-y}=\frac{1}{[1+(1-q) f(x)]} \frac{d f(x)}{d x},
$$

and its corresponding dual q-integral

$$
\int_{a}^{t} f(x) d^{q} x=\int_{a}^{t}[1+(1-q) f(x)] f(x) d x .
$$

The connection of q-derivative with the Hausdorff derivative was recently demonstrated[1]. It results that the relevant parameters may be connected as:

$$
1-q=\frac{(1-\varsigma)}{l_{0}}
$$

Consequently, one can see that the deformed q-derivative is the first order expansion of the Hausdorff derivative and that there is a strong connection between these formalisms by means of a fractal metric.

Recently, one of the authors (J.W.) has introduced what was named as scale-q- derivative [7]:

$$
D_{(q, \lambda)} f(\lambda x)=[1+(1-q) \lambda x] \frac{d f(\lambda x)}{d x},
$$


in such a way that the q-exponential with a scale $\lambda, e_{q}(\lambda x)$, is the eigenfunction of the differential equation

$$
[1+(1-q) \lambda x] \frac{d e_{q}(\lambda x)}{d x}=\lambda e_{q}(\lambda x)
$$

with eigenvalue $\lambda$, as the reader can verify. Here, $e_{q}(x)=[1+(1-q) x]^{\frac{1}{1-q}}$, is the $q$-exponential function that appears in the context of non extensive statistical mechanics [2].

\section{THE MODEL FOR SURVIVALFACTOR}

One of the advantages for the mapping to a fractal continuum is that the resulting model in this new space may take the random variable $x$ as a continuous variable, instead of a discrete one. The consideration of $D$ as a continuous variable was done also in Ref. [3].

Let $\mathrm{D}_{0}$ be the minimal annihilation dose [3], where no cell survive. Let us name the dimensionless dose as $x=\frac{D}{D_{0}}$.

To gain some insight, let us consider the simple model, considering the usual derivative.

Here, $N(x)$ is the number of cells at a given state of the radiation treatment when a dimensionless dose $x$ is achieved. Let us denote $\alpha$ as the dimensionless cell death probability after the achievement of dose $x$.

The model is analogous to the nuclear decay problem that uses time $t$ as the independent variable. Then, we can write:

$$
\frac{d N(x)}{d x}=-\alpha N(x)
$$

Note that $\alpha d x=p(x)$ is a constant probability density to kill a unitary cell by a radiation dose.

The well known solution of eq.(9) is $N(x)=N_{0} e^{-\alpha x}$. So, the survival factor, $\frac{N(x)}{N_{0}}=F_{S}$, can be written as $F_{S}=e^{-\alpha x}$, that reduces to the well known linear model in Ref. [35] if $\alpha=1$.

It is important to note that, since the survival factors $F_{S}$ are statistically independent, the doses $x$ are summable in the linear model [35], that means $F_{S}\left(x_{1}, x_{2}\right)=F_{S}\left(x_{1}+x_{2}\right)$. But in the context of the approach of complex systems, considering that tumors have complex structures, this implies that despite the $F_{S}$ are yet statistically independent, the doses must be here $q$-summable, due to non-related spaces. That is, $F_{S}\left(x_{1}, x_{2}\right)=F_{S}\left(x_{1} \oplus_{q} x_{2}\right)$, where $x_{1} \oplus_{q} x_{2}=x_{1}+x_{2}+(1-q) x_{1} x_{2}$. This justifies the approach of the mapping to a continuous fractal and consequently, the introduction of a structural derivative like scale-q-derivative.

Suppose now that we may proceed to a mapping to a fractal continuum due to a complex structure of the tumor cells and the derivative had to be redefined as a scale-q-derivative. The differential equation is now

$$
[1+(1-q) k x] \frac{d N(x)}{d x}=-\lambda N(x)
$$

that can also be considered as an equation that describes a non constant death probability, that is, a death probability that depends on the dose:

$$
p(x)=\frac{\lambda}{[1+(1-q) k x]} .
$$

The solution is

$$
\frac{N(x)}{N_{0}}=[1+(1-q) k x]^{-\frac{\lambda}{k(1-q)}}=\left[e_{q}(k x)\right]^{-\lambda / k} .
$$


Now, if we redefine variables as $k(1-q)=-1,-\frac{\lambda}{k(1-q)} \equiv \gamma$, that is nothing more than the model for survival factor in Ref. [3], $F_{S}=(1-x)^{\gamma}$. This model has excellent experimental proof in the literature [3, 4]. The linear-quadratic (LQ) model is recovered in the limit $q \rightarrow 1$ up to second order in a Taylor series expansion.

\section{SURVIVAL PROBABILITY}

In this section, we make some probabilistic arguments in order to reinforce the consistence of the models[3, 4].

Consider the cell death probability due to radiation absorbed as

$$
p(x)=\frac{\lambda}{(1-x)}
$$

that is the probability given in eq.(12) with $k(1-q)=-1$.

Consider now the escort probability, in such a way that it is defined in NESM as

$$
P(x)=\frac{p^{r}(x)}{\int p^{r}(x) d x}
$$

with normalized probability condition $\int_{0}^{1} P(x) d x=1$.

Where the entropic index is written here as $r$, in order to distinguish from the index $q$ used in the previous section. A simple calculation shows that, using eq.(14), the escort probability $P(x)$ can be written as

$$
P(x)=(1-r)(1-x)^{-r} .
$$

If we call $(1-r)=\gamma$, this leads to a death probability that depends on the dose as $\gamma(1-x)^{\gamma-1}$ and is similar to the result of Ref. [4], but here for radiation only. The main problem in this attempt to connect escort probability with the approach of structural derivatives is that the $r$ parameter here must be negative to give $\gamma>1$.

Independent of this lets try to obtain the factor $F_{S}$, in this context of NESM.

The survival factor can be seen as the probability difference from the probability of whole conformational space and the probability of cell death by radiation, that is,

$$
F_{S}=\left[1-\int_{0}^{x} P(x) d x\right]
$$

This can be rewritten as

$$
F_{S}=\int_{x}^{1} P(x) d x
$$

Again, a simple calculation leads to

$$
F_{S}=(1-x)^{1-r}=(1-x)^{\gamma},
$$

that is our the previous result for the survival factor.

Note that, if we redefine the $r$ parameter as $1-r=\frac{2-q}{1-q}=\gamma>1$, the result seems to be now compatible with that of Refs. [3, 4].

\section{CONCLUSIONS AND OUTLOOK}

In this contribution, we introduced, for the first time, the mathematical tool of structural derivatives to model the survival factor for tissues irradiation. 
The models are analogous to the nuclear decay problem, that takes the time, $t$, as the independent variable; here, instead, our independent variable is the applied dose, $x$.

We claim that the deformations of the derivative are the key to model complex systems; particularly here, we emphasize and focus on life, medical and biological science. Here, we adopt the local forms of derivative, called structural derivatives. However, we think that nonlocal forms of derivative, like fractional calculus, can help modeling too, but with much more computational and algebraic cost. We think that our model could help researchers to better understand models and dynamics, without excessive heuristics in the formulation.

As outlooks, we shall develop models with other forms of structural derivatives, in an attempt to achieve survive factors, that could be tested for the adequate kind of tumor cells. Also, the structural derivative may be applied to model earthquakes [36].

\section{ACKNOWLEDGMENTS}

The authors would like to thank J. A. Helayël-Neto for a critical reading of this article.

This work was performed under support of PRODEP project DSA/103.5/15/986 from SEP, Mexico.

\section{REFERENCES}

1. Weberszpil J., Lazo M. J. and Helayël-Neto J. A., Physica A 436, (2015) 399-404.

2. Borges E. P. , Physica A 340 (2004) 95-101.

3. Sotolongo-Grau O., Rodríguez-Pérez D., Antoranz J. C. and Sotolongo-Costa O., PRL 105, (2010) 158105-1158105-4.

4. Brouers F. and Sotolongo-Costa O., Physica A 368 (2006) 165-175.

5. Chen W. and Liang Y., International Conference on Fractional Differentiation and its Applications, Novi Sad, Serbia, July $18-20,2016$.

6. Chen W., Y. Liang and Hei X., Fractional Calculus and Applied Analysis, 195 (2016) 1250-1261.

7. Weberszpil, J. and Helayël-Neto, J.A., Physica. A (Print) 450, (2016) 217-227; arXiv:1511.02835 [math-ph].

8. Balankin A. S. and Espinoza B., Phys. Rev. E 85 (2012) 025302(R).

9. Balankin A. S. and Elizarraraz B. E., Phys. Rev. E 85, (2012) 056314.

10. Chen W., Chaos Solitons and Fractals 28 ( 2006) 923-929.

11. Abdeljawad T., Journal of Computational and Applied Mathematics 279 (2015) 57-66.

12. Rostamy D., ad Jabbari M., J. Appl. Environ. Biol. Sci., 2(4) (2012)159-171.

13. Benzekry S., Lamont C., Beheshti A.,Tracz A., Ebos J. M. L., Hlatky L. and Hahnfeldt P, PLoS Comput Biol 10(8) (2014) e1003800.

14. Buis R., Acta Biotheoretica 39 (1991) 185-195.

15. Caetano B., Cabella T. and Martinez A. S., Physical Review E 83 (2011) 061902.

16. Calderón C. P., Kwembe T. A., Mathematical Biosciences 103 (1991) 97-114.

17. Enderling H., Chaplain M. A., Curr Pharm Des. 20(30) (2014) 4934-40.

18. P. R. Koya, A. T. Goshu, Generalized Mathematical Model for Biological Growths, Open Journal of Modelling and Simulation, 1, 42-53, 2013.

19. Maruic M., Mathematical models of tumor growth, The lecture presented at the Mathematical Colloquium in Osijek organized by Croatian Mathematical Society - Division Osijek, June 7, 1996.

20. Sakanoue S., Ecological modelling, 2, 05 (2007) 159-168.

21. Souto M. A., González R. S. and Terçariol C. A. S., Physica A 387 (2008) 5679-5687.

22. Riewe F., Phys. Rev. E 53, (1996) 1890 ; ibid 55, (1997) 358.

23. Godinho C. F. L., Weberszpil J. and Helayël-Neto J. A., arXiv:1208.2266v3 [math-ph]; Int. J. Theor. Phys., 51, (2014) p.1.

24. Caldeira A. O. and Legett A. J., Phys. Rev. Lett. 46, (1981) 211; Ann. Phys. 149, (1983) 374; J. Ankerhold, H. Grabert and G. L. Ingold, Phys. Rev. E 51, (1995)4267. 
25. Senitzky I. R. , Phys. Rev. 119, 670 (1960); K. W. H. Stevens, Proc. Phys. Soc. London 72, (1958) 1027.

26. Golmankhaneh A. K. , Phys. Scr. (2009) 014008.

27. Lazo M. J., Conference Papers in Mathematics, v. 2013 (2013), Article ID 872869, 5p. doi:10.1155/2013/872869.

28. Weberszpil, J. and Helayël-Neto, J. A. . Advances in High Energy Physics, v. 2014, (2014) 1-12.

29. Zaslavsky G., Hamiltonian Chaos and Fractional Dynamics, Oxford University Press, New York, 2005.

30. Deppman A. Phys Rev. D 93 (2016) 054001.

31. Tarasov V. E., Adv. Ann. Phys. 318 (2005) 286.

32. Rajagopal A. K., Phys. Rev. Lett. 76 (1996) 3469.

33. Tsallis C., J. Stat. Phys. 52 (1988) 479.

34. Murray J.D. Mathematical Biology. Biomathematics Texts, vol. 19, Springer, 2nd ed., 1993.

35. Tubiana M., Introduction to Radiobiology,Taylor \& Francis, London, 1990.

36. Sotolongo-Costa O., Acta Geophysica vol. 60, no. 3 (2012), 526-534. 